\title{
Estimativa da disponibilidade de zinco em refeições com preparações padronizadas da alimentação escolar do município de Campinas
}

\author{
Estimated zinc availability in school meals done with \\ standard foods in the city of Campinas (SP), Brazil
}

Semíramis Martins Álvares DOMENE ${ }^{1}$

Thalita Cremonesi PEREIRA ${ }^{2}$

Rye Katsurayama de ARRIVILLAGA ${ }^{1}$

\section{RE S U M O}

\section{Objetivo}

Este estudo se propôs a avaliar razões molares fitato:Zn e fitatoxCa:Zn/MJ de preparações para escolares entre 7 e 14 anos.

\section{Métodos}

A padronização das receitas foi realizada em uma escola e submetida a teste de aceitação, por meio da avaliação do rejeito. As refeições habitualmente usadas foram submetidas à estimativa de disponibilidade de zinco por meio do cálculo das razões molares fitato:Zn e fitatoxCa:Zn/MJ. Os cálculos dietéticos foram realizados pelo programa Nut Win (versão 1.5) e foram usados os valores de 15 e 22 para as razões molares fitato: zinco e fitatoxCa:Zn/MJ, respectivamente, como pontos de corte para o risco à absorção de zinco.

\section{Resultados}

Os resultados mostram que 50,0\% das preparações apresentam risco à absorção de zinco segundo fitato: Zn, e 40,0\% segundo fitatoxCa:Zn/MJ; 82,0\% dos cardápios fornecem menos do que 15,0\% das recomendações de cálcio e 63,6\% apresentam grande probabilidade de inadequação de lipídeos.

\section{Conclusão}

A aplicação das razões molares para avaliação dos cardápios na alimentação escolar indica que a disponibilidade do zinco pode estar comprometida, o que pode ser revertido com o aumento da freqüência de alimentos fonte, como as carnes.

Termos de indexação: Ácido fítico. Alimentação escolar. Criança. Zinco.

\footnotetext{
1 Pontifícia Universidade Católica de Campinas, Centro de Ciências da Vida, Faculdade de Nutrição. Av. John Boyd Dunlop s/n., Campus II, Jd. Ipaussurama, 13060-904, Campinas, SP, Brasil. Correspondência para/Correspondence to: S.M.A. DOMENE.

2 Universidade Estadual de Campinas, Programa de Saúde da Criança e do Adolescente. Campinas, SP, Brasil.
} 
162 S.M.A. DOMENE et al.

\section{A B S T R A C T}

\section{Objective}

This study aimed to assess the molar ratios of phytate:Zn and phytatexCa:Zn/MJ in meals for school children aging from 7 to 14 years.

\section{Methods}

Recipes were standardized in a school and submitted an acceptance test by assessing meal leftovers. The availability of zinc in the usual meals was determined by calculating the molar ratios of phytate:zinc and phytatexCa:Zn/MJ. The dietary calculations were done with the program Nut Win (version 1.5) and 15 and 22 were used as cutoff values for the molar ratios of phytate:Zn and phytatexCa:Zn/MJ respectively for proper $\mathrm{Zn}$ absorption.

\section{Results}

The results show that $50.0 \%$ of the preparations present risk to the absorption of zinc according to the phytate: $\mathrm{Zn}$ ratio and $40.0 \%$ according to phytatex Ca: $\mathrm{Zn} / \mathrm{MJ}$ ratio; $82.0 \%$ of the diets offer less than $15.0 \%$ of the recommended calcium amounts and $63.6 \%$ are very likely to be inadequate regarding lipids.

\section{Conclusion}

The use of the molar ratios to assess the school menus indicates that zinc availability may be low. This could be reversed by serving good sources of zinc, such as meats, more often.

Indexing terms: Phytic acid. School feeding. Children. Zinc.

\section{N T R O D U Ç Ã O}

O Programa Nacional de Alimentação Escolar (PNAE), que atendeu a 36 milhões de crianças no ano de 2002, completou 50 anos em 2005 e tem metas complementares à agenda nacional de políticas públicas na área de nutrição, que ganharam força com a proposta do Programa Fome Zero ${ }^{1}$. Dada a cobertura nacional, o PNAE assume características próprias que divergem em cada região do Brasil, sendo que o município ajusta a gestão local de acordo com suas particularidades. Este Programa visa a atender escolas públicas, filantrópicas e indígenas e tem como objetivo suplementar o dia alimentar da criança fornecendo, no mínimo, 15\% das necessidades nutricionais, contribuir para a redução da evasão escolar e favorecer a formação de bons hábitos alimentares em crianças e adolescentes do País ${ }^{2}$.

De acordo com o sistema de gestão municipalizada, o financiamento proveniente do Fundo Nacional de Desenvolvimento da Educação (FNDE) é repassado diretamente à Prefeitura Municipal de Campinas (PMC), que, tradicionalmente, complementa este valor para melhor atendimento às metas do Programa. A PMC tem a função de administrar o Programa de Alimentação Escolar na cidade, contando com a parceria da Central de Abastecimento de Campinas S.A. (CEASA), desde o ano de 2001, sendo esta a responsável pela elaboração de cardápios para a alimentação escolar e pré-escolar. Um dos projetos de melhoria da alimentação escolar na cidade de Campinas, que está em andamento, é a padronização de cardápios, que conta com a colaboração da Faculdade de Nutrição da Pontifícia Universidade Católica de Campinas (PUC-Campinas). Este projeto visa a subsidiar ações que permitam a elaboração de preparações nutricionalmente equilibradas para escolares entre 7 e 14 anos, com prioridade de oferta de alimentos in natura, de forma que o custo seja compatível com o orçamento gerado pelo repasse do Fundo Nacional para o Desenvolvimento da Educação (FNDE), e pela complementação do poder público local. A gestão municipal do PNAE, que estabelece parcerias com este projeto e com outras iniciativas, como o treinamento de merendeiras, foi premiada pelo Prêmio Gestor Eficiente da Merenda Escolar no ano de $2004^{3}$. 
Uma das formas de verificar a qualidade de cardápios é estimar a biodisponibilidade dos nutrientes presentes nas preparações, o que pode ser feito por meio do cálculo de razões molares fitato:zinco (fitato:Zn). O Zinco (Zn) é um mineral envolvido em passos metabólicos relevantes para o bom funcionamento do sistema imune e para o crescimento. Sua absorção intestinal pode ser prejudicada pela ação do fitato naturalmente presente nos alimentos, neste caso, desempenhando ação antinutricional, dada sua capacidade de ligação a cátions mono e divalentes, diminuindo sua solubilidade intra-luminal ${ }^{4}$. Este efeito inibitório é potencializado quando há cálcio (Ca) na refeição ${ }^{5}$. Está demonstrado que razões molares fitato:Zn e fitatoxCa:Zn/MJ superiores, respectivamente, aos valores de 15 e 22 nas preparações, são indicadores de menor absorção de zinco e, conseqüentemente, limitadoras de sua biodisponibilidade ${ }^{6,7}$.

É evidente na literatura a relação entre dietas com razões molares fitato:Zn e fitatoxCa:Zn superiores aos valores de risco com efeitos sobre a saúde de crianças, como baixa concentração de Zn capilar, menor absorção de Zn e influência negativa no crescimento ${ }^{8-10}$. O fitato, especialmente em sua forma hexafosfórica, exerce importante efeito quelante sobre os cátions da dieta em condições de $\mathrm{pH}$ alcalino, como o observado no meio intraluminal. A estimativa da biodisponibilidade, portanto, depende da composição de todos os alimentos consumidos na mesma refeição'11.

Este estudo tem como objetivo avaliar o valor nutricional e estimar a biodisponibilidade de Zn de preparações que estão sendo padronizadas para a alimentação escolar da cidade de Campinas, considerando as refeições em que são servidas.

\section{M É T O D O S}

Realizou-se cálculo dietético por meio do Programa de Apoio à Nutrição Nut Win (versão 1.5) dos cardápios compostos por 11 preparações que estão sendo padronizadas para atender aos escolares de 7 a 14 anos de idade. O fornecimento estimado de nutrientes foi comparado com as recomendações norte-americanas levando-se em consideração que a alimentação escolar deve prover $15 \%$ das necessidades de todos os nutrientes ${ }^{12-15}$. Para a energia empregou-se o valor médio de recomendação (Estimated Energy Requirement - EER) para o estágio de vida correspondente. Para lipídeos e carboidratos utilizou-se o intervalo estabelecido pelo Acceptable Macronutrient Distribution Ranges (AMDR).

Realizou-se o cálculo das razões molares fitato:Zn e fitatoxCa:Zn/MJ destas preparações para estimar a biodisponibilidade de Zn, tendo-se obtido os teores de fitato nos alimentos por meio de compilação da literatura' ${ }^{16-19}$.

\section{RESULTADOS}

A Tabela 1 mostra os valores das razões molares de cada porção estudada, além da média (M) e do desvio-padrão (DP) entre as refeições. Consideraram-se, para o cálculo da média e do DP, apenas 10 preparações, excluindo-se aquelas cujos teores de fitato encontrados na literatura mostram ser este conteúdo inexpressivo nos ingredientes empregados em sua formulação, ou não foram encontrados. As médias de fitato:Zn

Tabela 1. Razões molares fitato:Zn e fitatoxCa:Zn/MJ das refeições empregadas no Programa Nacional de Alimentação Escolar em Campinas. Campinas (SP), 2005.

\begin{tabular}{|c|c|c|}
\hline Preparação & Fitato:Zn & FitatoxCa:Zn/MJ \\
\hline Sopa de legumes com macarrão ${ }^{6}$ & 7,25 & 6,26 \\
\hline Canja de frango ${ }^{6}$ & 21 & 11,78 \\
\hline Caldo enriquecido 6 & 7 & 5,5 \\
\hline Carne com abóbora ${ }^{4}$ & $\mathrm{nd}^{*}$ & $n d^{*}$ \\
\hline Bolo de banana 5 & 16 & 10,68 \\
\hline Arroz à primavera & 3 & 1,68 \\
\hline Carne moída com legumes ${ }^{1}$ & 15 & 16 \\
\hline Doce de abóbora² & 5 & 25,5 \\
\hline Doce de banana ${ }^{2}$ & 5 & 24,3 \\
\hline Frango xadrez ${ }^{1}$ & 22,5 & 37,3 \\
\hline \multirow[t]{3}{*}{ Patê de berinjela ${ }^{3}$} & 50 & 74,5 \\
\hline & $\mathrm{DP}$ & DP \\
\hline & $15,18 \quad 14,1$ & $21,35 \quad 21,6$ \\
\hline
\end{tabular}

* nd= valor não determinado; Acompanhamentos: ${ }^{1}$ arroz e feijão; ${ }^{2}$ pão e leite com achocolatado; ${ }^{3}$ pão e suco; ${ }^{4}$ arroz; ${ }^{5}$ suco; ${ }^{6}$ maçã. 
164 | S.M.A. DOMENE et al.

e fitatoxCa:Zn/MJ encontradas foram de 15 e 21, respectivamente. Cerca de $50 \%$ das preparações apresentaram valores de fitato: $Z n$ de risco à absorção de Zn (>15), e a freqüência de $40 \%$ foi encontrada para a razão fitatoxCa:Zn/MJ de risco (>22).

Na Tabela 2 estão os resultados do cálculo dietético das porções individuais das preparações quanto a energia, macronutrientes, Zn e Ca, acompanhados dos valores de Média e Desvios-padrão.

A ocorrência de elevados valores de desvios-padrão para o Ca ilustra a diversificação da freqüência do uso de alimentos fontes deste nutriente em cada preparação.
As freqüências de adequação do atendimento das recomendações nutricionais para energia e macronutrientes constam na Tabela 3, e para Zn e Ca na Tabela 4.

Todas as preparações apresentam grande probabilidade de adequação quanto a proteínas e carboidratos. Aproximadamente metade das preparações tem grande probabilidade de adequação de energia e zinco. Entretanto, há muitas preparações com alta probabilidade de inadequação de lipídeos e $\mathrm{Ca}$, segundo os dados apresentados nas Tabelas 3 e 4 .

Tabela 2. Valor energético, de macronutrientes, Zn e Ca, de porções individuais das refeições empregadas no Programa Nacional de Alimentação Escolar em Campinas. Campinas (SP), 2005.

\begin{tabular}{|c|c|c|c|c|c|c|c|c|c|c|}
\hline Preparação & Energia (Kcal) & $P(g)$ & \multicolumn{2}{|c|}{$\mathrm{L}(\mathrm{g})$} & \multicolumn{2}{|c|}{$\mathrm{CHO}(\mathrm{g})$} & \multicolumn{2}{|c|}{$\mathrm{Zn}(\mathrm{mg})$} & \multicolumn{2}{|c|}{$\mathrm{Ca}(\mathrm{mg})$} \\
\hline Sopa de legumes com macarrão ${ }^{6}$ & 231,1 & 10,0 & \multicolumn{2}{|c|}{3,9} & \multicolumn{2}{|c|}{41,3} & \multicolumn{2}{|c|}{1,12} & \multicolumn{2}{|c|}{32,8} \\
\hline Canja de frango ${ }^{6}$ & 265,5 & 9,8 & \multicolumn{2}{|c|}{2,7} & \multicolumn{2}{|c|}{51,9} & \multicolumn{2}{|c|}{0,77} & \multicolumn{2}{|c|}{27,9} \\
\hline Caldo enriquecido ${ }^{6}$ & 299,5 & 8,2 & \multicolumn{2}{|c|}{8,9} & \multicolumn{2}{|c|}{49,9} & \multirow{2}{*}{\multicolumn{4}{|c|}{$\begin{array}{l}40,7 \\
12,0\end{array}$}} \\
\hline Carne com abóbora ${ }^{4}$ & 212,9 & 9,8 & \multicolumn{2}{|c|}{1,8} & \multicolumn{2}{|c|}{37,9} & & & & \\
\hline Bolo de banana ${ }^{5}$ & 267,3 & 6,4 & \multicolumn{2}{|c|}{2,9} & \multicolumn{2}{|c|}{54,7} & \multicolumn{4}{|c|}{7,7} \\
\hline Arroz à primavera & 308,8 & 10,3 & \multicolumn{2}{|c|}{13,0} & \multicolumn{2}{|c|}{36,9} & & & & 0 \\
\hline Carne moída com legumes ${ }^{1}$ & 339,0 & 13,3 & & & & & & & &, 5 \\
\hline Doce de abóbora² & 371,9 & 13,4 & & & & & & & & \\
\hline Doce de banana ${ }^{2}$ & 384,1 & 13,4 & & & & & & & & \\
\hline Frango xadrez ${ }^{1}$ & 323,1 & 16,4 & & & & & & & & 4 \\
\hline Patê de berinjela ${ }^{3}$ & 252,0 & 5,3 & & & & & & & & 3 \\
\hline & DP & M & M & DP & M & DP & M & DP & M & $\mathrm{DP}$ \\
\hline & 296 & 11 & 6,4 & 3,8 & 49,7 & 8,1 & 1,1 & 0,58 & 89 & 113,4 \\
\hline
\end{tabular}

Acompanhamentos: ${ }^{1}$ arroz e feijão; ${ }^{2}$ pão e leite com achocolatado; ${ }^{3}$ pão e suco; ${ }^{4}$ arroz; ${ }^{5}$ suco; ${ }^{6}$ maçã; M: média; DP: desvio-padrão.

Tabela 3. Freqüência de adequação da energia e dos macronutrientes das porções individuais de cada preparação. Programa Nacional de Alimentação Escolar. Campinas (SP), 2005.

\begin{tabular}{|c|c|c|c|c|c|c|c|c|c|c|c|c|c|c|c|}
\hline \multicolumn{4}{|c|}{ Energia $^{1}$} & \multicolumn{4}{|c|}{ Proteína ${ }^{1}$} & \multicolumn{4}{|c|}{ Lipídeos $^{1}$} & \multicolumn{4}{|c|}{ Carboidratos $^{1}$} \\
\hline \multicolumn{2}{|c|}{ Valores $<$ EER } & \multicolumn{2}{|c|}{ Valores >EER } & \multicolumn{2}{|c|}{ Valores < Al } & \multicolumn{2}{|c|}{ Valores >Al } & \multicolumn{2}{|c|}{ Valores <AMDR } & \multicolumn{2}{|c|}{ Valores >AMDR } & \multicolumn{2}{|c|}{ Valores < AMDR } & \multicolumn{2}{|c|}{ Valores >AMDR } \\
\hline$n$ & $\%$ & $n$ & $\%$ & $n$ & $\%$ & $n$ & $\%$ & $n$ & $\%$ & $\mathrm{n}$ & $\%$ & $n$ & $\%$ & $n$ & $\%$ \\
\hline 5 & 45,5 & 6 & 54,5 & 0 & 0 & 11 & 100 & 7 & 63,6 & 4 & 36,4 & 0 & 0 & 11 & 100 \\
\hline
\end{tabular}

${ }^{1}$ Considerando $15 \%$ das recomendações nutricionais, conforme legislação em vigor. EER: estimated energy requirement; Al: adequate intakes; AMDR: acceptable micronutrient distribution ranges.

Tabela 4. Freqüência de adequação de Zinco e Cálcio das porções individuais de cada preparação. Programa Nacional de Alimentação Escolar. Campinas (SP), 2005.

\begin{tabular}{|c|c|c|c|c|c|c|c|c|c|}
\hline \multicolumn{4}{|c|}{$\mathrm{Ca}^{1}$} & \multicolumn{6}{|c|}{$\mathrm{Zn}{ }^{1}$} \\
\hline \multicolumn{2}{|c|}{ Valores <Al } & \multicolumn{2}{|c|}{ Valores >Al } & \multicolumn{2}{|c|}{ Valores $<$ EAR } & \multicolumn{2}{|c|}{ EAR $<$ Valores $<$ RDA } & \multicolumn{2}{|c|}{ Valores $>$ RDA } \\
\hline$n$ & $\%$ & $\mathrm{n}$ & $\%$ & $\mathrm{n}$ & $\%$ & $n$ & $\%$ & $n$ & $\%$ \\
\hline 9 & 81,8 & 2 & 18,2 & 5 & 45,5 & 1 & 9 & 5 & 45,5 \\
\hline
\end{tabular}

${ }^{1}$ Considerando $15 \%$ das recomendações nutricionais, conforme legislação em vigor. Al: adequate intakes; EAR: estimated average requirement; RDA: recommended dietary allowances. 


\section{DISCUSS Ã O}

Com a análise dos resultados observa-se que as médias das razões molares fitato:Zn e fitatoxCa:Zn/MJ não ultrapassam os valores de risco de 15 e 22, respectivamente. Entretanto, as médias são muito próximas ao valor de risco, e $50 \%$ das 10 preparações estudadas estão acima de 15 para fitato:Zn, e 40\% estão acima de 22 para fitatoxCa:Zn/MJ. Tais freqüências de razões molares de risco apresentam-se superiores à freqüência encontrada em refeições de jovens e adultos da Escócia ${ }^{20}$.

Entre as causas da alta freqüência de razões molares de risco entre as preparações está a utilização de ingredientes e alimentos com elevado conteúdo fitato para refeições pobres em alimentos de origem animal, como arroz branco, farinha de trigo refinada, feijão e pão branco. A existência de alimentos predominantemente vegetais determina baixo fornecimento de zinco em 6 das 11 preparações, prejudicando, assim, a sua biodisponibilidade. $\mathrm{O}$ aumento do consumo de alimentos de origem animal como estratégia para melhorar a biodisponibilidade de micronutrientes é um recurso empregado por outros autores. Em uma comunidade da Malásia, Gibson et al. ${ }^{21}$ observaram maior ingestão de micronutrientes em grupo de crianças que recebeu $8,9 \mathrm{~g}$ de proteína de origem animal por dia, em relação ao grupo que recebeu $5,1 \mathrm{~g}$, com diminuição da prevalência de anemia e doenças infecciosas. Contudo, os autores alertam para o fato de que, sendo o peixe a fonte protéica empregada no estudo, o consumo de ferro não foi afetado. 0 baixo consumo de alimentos de origem animal foi apontado como uma das principais características da dieta de crianças quenianas de 8 a 9 anos, sendo que neste grupo a prevalência de anemia foi de $50 \%$.

Deve-se lembrar que em uma das preparações não foi possível estimar a biodisponibilidade de Zn, pela ausência de dados na literatura quanto ao valor de fitato. Esta preparação foi desconsiderada para o cálculo das médias das razões molares e da freqüência de risco à absorção do Zn. Este exemplo ilustra a importância da elaboração de uma base de dados com o teor de ácido fítico nos alimentos, obtidos por meio de análises laboratoriais eficientes e confiáveis.

Parece que a quantidade de Ca nas refeições apresentadas não é fator preocupante quanto ao efeito sobre a biodisponibilidade de Zn, pois $81,8 \%$ dessas preparações não oferecem adequado aporte de $\mathrm{Ca}$, diminuindo a chance de ligação ao fitato e assim, não aumentando a razão molar fitatoxCa:Zn/MJ. Contudo, esta situação de baixa ingestão de Ca é preocupante, pois a formação do material ósseo é predominante na infância e demanda uma adequada ingestão do mineral em questão ${ }^{23}$. As únicas preparações que ofereceram quantidade adequada de Ca foram aquelas que possuíam como acompanhamento o leite achocolatado.

O fato de que a alimentação escolar em estudo não fornece $15 \%$ das necessidades nutricionais, na maioria dos dias da semana, já foi apontado anteriormente ${ }^{24}$. Existem poucos trabalhos com dados de outros municípios que permitam estabelecer comparações. O baixo fornecimento de micronutrientes na alimentação escolar está confirmado no presente estudo, por meio das porcentagens com maior probabilidade de inadequação de Zn e Ca. Entretanto, quanto a energia e proteínas, diverge da pesquisa citada, uma vez que a maioria das preparações analisadas em neste estudo apresentou alta probabilidade de adequação; isto pode decorrer do ajuste recente dos valores de referência para recomendações energéticas $^{14}$.

Todas as preparações se ajustaram às recomendações de proteína e carboidratos, oferecendo $15 \%$ ou mais das necessidades dos escolares. Já a energia teve valor menor que $15 \%$ das recomendações em 5 das preparações, provavelmente devido à inadequação de lipídeos, que teve grande probabilidade de inadequação em 7 preparações. É necessário que a alimentação escolar forneça a quantidade adequada de energia para que se minimize a possibilidade, demonstrada na pesqui- 
166 S.M.A. DOMENE et al.

sa de Mazzilli25, de que as crianças apresentem deficiência de consumo energético no restante do seu dia alimentar. Entretanto, o padrão da refeição fornecida na época do estudo de Mazzilli25 induzia a supressão da refeição subseqüente, uma vez que era constituído, comumente, por preparações de elevada densidade energética, especialmente produtos formulados, felizmente em desuso pela atual proposta do PNAE.

\section{O N CLUS Ã O}

Os dados deste estudo mostram que $\mathrm{O}$ consumo das preparações oferecidas pelo PNAE aos escolares de Campinas não atende rigorosamente à previsão quantitativa de nutrientes. Isto implica em aporte energético e nutricional insuficientes, devido ao viés da demanda espontânea de alimentos consumidos. Por isto é importante que, no momento da elaboração dos cardápios para a alimentação escolar, deva-se atentar para a adequada previsão de fornecimento de nutrientes, e com misturas alimentares que promovam a absorção de micronutrientes, especialmente de $Z n$, para que as razões molares fitato:Zn e fitatoxCa:Zn/MJ não ultrapassem o valor de risco para a absorção do mineral. Além disso, é necessário fornecer mais alimentos ricos em Ca em cada preparação, já que houve grande probabilidade de inadequação em muitas das preparações estudadas. Uma maior quantidade de Ca somente irá potencializar o efeito inibitório do fitato com o Zn, se este último mineral apresentar-se em quantidades inadequadas nas preparações ou, ainda, se forem fornecidos grãos integrais nos cardápios dos escolares, o que seria contra-indicado com os atuais níveis de fornecimento de alimentos de origem animal.

É necessário atentar para outros aspectos observados no presente trabalho, como a pequena quantidade de lipídeos, fontes de ácidos graxos insaturados indispensáveis, a fim de que estes sejam fornecidos em teores satisfatórios, considerando o dia alimentar da criança. Este fato é relevante em preparações de refeição em grande escala, quando o rendimento do óleo é maior do que o observado em pequenas proporções, resultando em quantidades menores nos processos de padronização de receitas.

Sugere-se que as refeições a serem empregadas no Programa de Alimentação Escolar sejam anteriormente submetidas a comparações com as novas recomendações nutricionais, para que o objetivo do PNAE seja efetivado.

\section{A GRADECIMENTOS}

À Central de Abastecimento de Campinas S.A. (CEASA), por ter disponibilizado as receitas das preparações, ao Conselho Nacional de Desenvolvimento Científico e Tecnológico (CNPq) pela bolsa de Iniciação Científica e à Fundação de Amparo à Pesquisa do Estado de São Paulo (FAPESP), pelo apoio ao projeto (Processo 98/14020,-1).

\section{COLABORADORES}

S.M.A. DOMENE participou da concepção, da coleta de dados, da análise e da redação do artigo. T.C. PEREIRA e R.K. ARRIVILLAGA participaram da coleta de dados, da análise e da redação do artigo.

\section{REFERÊ NCIAS}

1. Furtuoso MCO, Ometto $A M H$, Pipitone MAP, Silva MV, Sturion GL. Fatores condicionantes da adesão dos alunos ao Programa de Alimentação Escolar no Brasil. Rev Nutr. 2005; 18(2):167-81.

2. Fundo Nacional de Desenvolvimento da Educação. Resolução $n^{\circ} 35$, de 1 de outubro de 2003. Estende atendimento do PNAE a creches e estabelece valores per capita. [acesso 2005 ago 9]. Disponível em: <http://www.fnde.gov.br/home/legislacao _manuais/alimentacao_escolar/res035 01102003.pdf>.

3. Apoio Fome Zero. Prêmio Gestor Eficiente da Merenda Escolar. [acesso 2005 ago 19]. Disponível em: <http://www.apoiofomezero.org.br/site/ index.asp?Fuseaction $=$ Conteudo\&ParentID $=45 \&$ \& Menu=45\&Materia $=212>$.

4. Mazariegos M, Hambidge KM, Krebs NF, Westcott JE, Lei S, Grunwald GK, et al. Zinc absorption in Guatemalan schoolchildren fed normal or 
low-phytate maize. Am J Clin Nutr. 2006; 83(1): 59-64.

5. Hambidge KM, Krebs NF, Westcott JL, Sian L, Miller $\mathrm{LV}$, Peterson $\mathrm{KL}$, et al. Absorption of calcium from tortilla meals prepared from low-phytate maize. Am J Clin Nutr. 2005; 82(1):84-7.

6. Davies NT, Olpin SE. Studies on the phytate: zinc molar contents in diets as a determinant of $\mathrm{Zn}$ availability to young rats. Br J Nutr. 1979; 41: 591-603.

7. Fordyce EJ, Forbes RM, Robbins KR, Erdman JW Jr. Phytate $\mathrm{x}$ calcium/zinc molar ratios: are they predictive of zinc bioavailability? J Food Sci. 1987; 52:440-4.

8. Broadhead RL, Gibson RS, Hambidge KM, Hotz C, Krebs NF, Manary MJ, et al. Zinc homeostasis in Malawian children consuming a high-phytate, maize-based diet. Am J Clin Nutr. 2002; 75(6): 1057-61.

9. Ferguson EL, Gibson RS, Opare-Obisaw C, Ounpuu S, Thompson LU, Lehrfeld J. The zinc nutriture of preschool children living in two African countries. J Nutr. 1993; 123(9):1487-96.

10. Gibson RS, Vanderkooy S, Thompson L. Dietary phytate $\mathrm{x}$ calcium/zinc millimolar ratios and zinc nutriture in some Ontario preschool children. Biol Trace Elem Res. 1991; 30(1):87-94.

11. Haraldsson AK, Veide J, Andlid T, Alminger ML, Sandberg AS. Degradation of phytate by high-phytase Saccharomyces cerevisiae strains during simulated gastrointestinal digestion. J Agric Food Chem. 2005; 53(13):5438-44.

12. Institute of Medicine. National Academy of Sciences on Dietary Reference Intakes (DRI). Dietary reference intakes: applications in dietary assessment. Washington (DC): National Academy Press; 2001.

13. Institute of Medicine. National Academy of Sciences on Dietary Reference Intakes (DRI). Dietary reference intakes for calcium, phosphorus, magnesium, vitamin D, and fluoride. Washington (DC): National Academy Press; 1999.

14. Institute of Medicine. National Academy of Sciences on Dietary Reference Intakes (DRI). Dietary reference intakes for energy, carbohydrate, fiber, fat, fatty acids, cholesterol, protein, and amino acids (macronutrients) pre-publication. Washington (DC): National Academy Press; 2002.
15. Institute of Medicine. National Academy of Sciences on Dietary Reference Intakes (DRI). Dietary reference intakes for vitamin A, vitamin K, arsenic, boron, chromium, copper, iodine, iron, manganese, molybdenum, nickel, silicon, vanadium, and zinc. Washington (DC): National Academy Press; 2002.

16. Adams CL, Dorsch JA, Hambidge M, Krebs NF, Raboy $\mathrm{V}$, Sian L, et al. Zinc absorption from a low-phytic acid maize. Am J Clin Nutr. 2002; 76(3):556-9.

17. Cardoso SP, Martins C. Interações droga-nutriente. Curitiba: NutroClínica; 1998.

18. Hulthén LR, Larsson M, Sandberg AS, Sandstrom B. Improved zinc and iron absorption from breakfast meals containing malted oats with reduced phytate content. Br J Nutr. 1996; 76: 677-88.

19. Oberleas D, Harland BE. Phytate content of foods: effect on dietary zinc bioavailability. J Am Diet Assoc. 1981; 79:433-6.

20. Liddell J, Lockie GM, Wise A. Dietary intakes of phytate and its meal distribution pattern amongst staff and students in an institution of higher education. Br J Nutr. 1987; 58(3):337-46.

21. Gibson RS, Yeudall F, Drost N, Mtitimuni BM, Cullinan TR. Experiences of a community-based dietary intervention to enhance micronutrient adequacy of diets low in animal source foods and high in phytate: a case study in rural Malawian children. J Nutr. 2003; 133(11 Suppl 2):3992S-9S.

22. Bwibo NO, Neumann CG. The need for animal source foods by Kenyan children. J Nutr. 2003; 133(11 Suppl 2):3936S-40S.

23. Krebs NF. Bioavailability of dietary supplements and impact of physiologic state: infants, children and adolescents. J Nutr. 2001; 131(suppl 4):1351-54.

24. Tereso MJA, Vianna RPT. O Programa de Merenda Escolar de Campinas: análise do alcance e limitações do abastecimento regional. Rev Nutr. 2000; 13(1):41-9.

25. Mazzilli RN. Merenda no dia alimentar de crianças matriculadas em centros de educação e alimentação do pré-escolar. Rev Saúde Pública. 1987; 21(4):317-25.

Recebido em: 8/3/2007

Versão final reapresentada em: 8/3/2008 Aprovado em: 21/2/2008 